\title{
A new therapy (MP29-02*) provides effective relief from all individual nasal and ocular symptoms of seasonal allergic rhinitis
}

\author{
Peter Hellings ${ }^{1 *}$, Claus Bachert ${ }^{2}$, Jean Bousquet ${ }^{3}$, Glenis Scadding ${ }^{4}$, Wytske Fokkens ${ }^{5}$, Ullrich Munzel $^{6}$, David Price $^{7}$ \\ From 9th Symposium of Experimental Rhinology and Immunology of the Nose (SERIN 2013) \\ Leuven, Belgium. 21-23 March 2013
}

\section{Background}

Congestion is reported by allergic rhinitis (AR) patients as the most bothersome nasal symptom. However, ocular symptoms have the greatest impact on patient quality of life. A novel treatment which more effectively controls both nasal and ocular symptoms would fill the gap for an important unmet need in AR.

\section{Objective}

To assess the efficacy of MP29-02* (a novel intranasal formulation of azelastine hydrochloride [AZE] and fluticasone propionate [FP]) in providing relief from each of the nasal and ocular symptoms commonly experienced by patients with seasonal AR (SAR) compared to commercially available intranasal AZE or FP nasal sprays or placebo.

\section{Methods}

Patients ( $\geq 12$ years old) with moderate-to-severe SAR $(\mathrm{n}=610)$ were randomized into this double-blind, placebo-controlled, 14-day, parallel-group trial to MP29$02^{*}$, commercially-available AZE or FP nasal sprays, or placebo (all given as 1 spray/nostril bid [total daily dose:

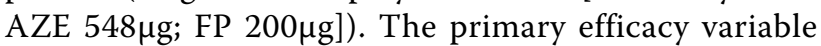
was change from baseline in reflective total nasal symptom score (rTNSS; AM +PM) over 14-days. Secondary endpoints included change from baseline in each of the individual nasal and ocular symptoms.

\section{Results}

MP29-02* reduced nasal congestion by -1.24 vs -0.86 for FP (Diff: $-0.39 ; 95 \%$ CI $-0.65,-0.13 ; p=0.0034$ ), -0.75 for AZE (Diff: $-0.49 ; 95 \%$ CI: $-0.74,-0.24 ; \mathrm{p}=0.0001$ ) and -0.54 for placebo (Diff: -0.70 ; $95 \%$ CI $-0.95,-0.45 ; \mathrm{p}<0.0001$ ),

${ }^{1}$ University Hospitals Leuven, Leuven, Belgium

Full list of author information is available at the end of the article with a relative difference of $54 \%$ to FP and $70 \%$ to AZE. A similar and significant superiority of MP29-02* over FP and AZE was observed for nasal itching (44\% to FP, 56\% to AZE), rhinorrhoea (32\% to FP; $65 \%$ to AZE) and sneezing (49\% to FP; $61 \%$ to AZE). The most bothersome ocular symptom of itching was reduced by -1.23 by MP29-02* vs -0.70 for FP (Diff: -0.53 ; 95\% CI: $-0.79,-0.26$; $\mathrm{p}=0.0001$ ), -0.88 for AZE (Diff: -0.35 ; $95 \%$ CI $-0.63,-0.08 ; \mathrm{p}=0.0127$ ) and -0.44 for placebo (Diff: $-0.79,95 \%$ CI $-1.04,-0.54$; $\mathrm{p}<0.0001$ ) with a relative difference of $67 \%$ to FP and $44 \%$ to AZE. The relative differences to FP and AZE respectively were $51 \%$ and $25 \%$ for ocular watering, and $53 \%$ and $40 \%$ for ocular redness.

\section{Conclusion}

MP29-02* provides superior relief from each nasal and ocular symptom compared to first line AR therapies, including the most bothersome symptoms of nasal congestion and ocular itching, and can be considered the drug of choice for AR.

"Dymista

\section{Author details}

University Hospitals Leuven, Leuven, Belgium. ${ }^{2}$ Ghent University Hospital, Dept of Oto-rhinolaryngology, Ghent, Belgium. ${ }^{3}$ Hopital Arnaud de Villeneuve University Hospital, Montpellier, France. ${ }^{4}$ The Royal National Throat, Nose and Ear Hospital, London, UK. ${ }^{5}$ Academic Medical Center, Dept of Otorhinolaryngology, Amsterdam, the Netherlands. ${ }^{6}$ Meda Pharma, Biostatistics \& Market Access, Bad Homburg, Germany. ${ }^{7}$ Univserity of Aberdeen, Dept of General Practice \& Primary Care, Aberdeen, UK.

Published: 16 July 2013

\section{doi:10.1186/2045-7022-3-S2-P41}

Cite this article as: Hellings et al:: A new therapy (MP29-02*) provides effective relief from all individual nasal and ocular symptoms of seasonal allergic rhinitis. Clinical and Translational Allergy 2013 3(Suppl 2): P41. 